PROCEEDINGS OF THE

AMERICAN MATHEMATICAL SOCIETY

Volume 131, Number 10, Pages 3143-3154

S 0002-9939(03)06859-X

Article electronically published on February 12, 2003

\title{
ON VON NEUMANN'S PROBLEM IN EXTENSION THEORY OF NONNEGATIVE OPERATORS
}

\author{
YURY ARLINSKIĬ AND EDUARD TSEKANOVSKII
}

(Communicated by Joseph A. Ball)

\begin{abstract}
The solution of von Neumann's problem about parametrization of all nonegative selfadjoint extensions of a nonnegative densely defined operator in terms of his formulas is obtained.
\end{abstract}

1.

John von Neumann [18 formulated a problem about existence and description (parametrization) of all selfadjoint extensions preserving the lower bound of a given densely defined symmetric operator bounded from below acting on some Hilbert space. The existing descriptions of all nonnegative self-adjoint extensions of a nonnegative densely defined operator are not in terms of von Neumann's classical formulas. They have been obtained indirectly by M.Kreln [15], 16] in terms of his theory of self-adjoint contractive extensions of non-densely defined Hermitian contractions and by M.Birman [6] for a symmetric operator with strictly positive lower bound using the M.Vishik approach [20] (see [1]). In terms of abstract boundary conditions 13 and the Weyl-Titchmarsh functions 8], 11, 12, descriptions of the domains of all nonnegative self-adjoint and proper $m$-sectorial extensions were obtained in 9 .

In this paper, taking into account methods and approaches in [3, 4, [17, we establish new formulas (Theorem 2) which provide a parametrization of all nonnegative self-adjoint extensions of a nonnegative symmetric operator with, generally speaking, zero lower bound under the assumption that the so-called Friedrichs extension [15] of this operator is known. As a result of this approach we obtain a solution of von Neumann's problem about parametrization of all nonnegative selfadjoint extensions in terms of his formulas. Since the Friedrichs extension can be found independently of the above-mentioned methods, it turns out that the presented approach is efficient. An example of an operator with zero lower bound is considered. We plan to consider applications to canonical resolvents, pointinteractions in $\mathbb{R}^{3}$ with $m$-points of interaction, perturbation theory and the theory of Krein-Langer $Q$-functions in a future paper.

We shall use the following notations: $\mathcal{L}\left(H_{1}, H_{2}\right)$ denotes the Banach space of all continuous linear operators acting from the Hilbert space $H_{1}$ into the Hilbert

Received by the editors August 6, 2001 and, in revised form, May 6, 2002.

2000 Mathematics Subject Classification. Primary 47A63, 47B25; Secondary 47B65.

(C)2003 American Mathematical Society 
space $H_{2}, \mathcal{L}(H)=\mathcal{L}(H, H)$, and $\mathcal{D}(T), \mathcal{R}(T)$, Ker $T, \rho(T)$ denote the domain, the range, the null-space and the resolvent set of a linear operator $T$, respectively.

2 .

Let $H$ be a complex Hilbert space and let $H^{2}$ be the Hilbert space of all pairs $\left\langle u_{1}, u_{2}\right\rangle, u_{1}, u_{2} \in H$, with the inner product defined by

$$
\left(\left\langle u_{1}, u_{2}\right\rangle,\left\langle v_{1}, v_{2}\right\rangle\right)=\left(u_{1}, v_{1}\right)+\left(u_{2}, v_{2}\right) .
$$

As is well known [7], a closed subspace $\mathbf{T} \subseteq H^{2}$ is called a linear relation (l.r.) in $H$. We denote by $\mathcal{D}(\mathbf{T})$ the domain of a linear relation $\mathbf{T}$ and by $\mathbf{T}(u)$ the set of all $v$ such that $\langle u, v\rangle \in \mathbf{T}$. By definition

$$
\begin{aligned}
\mathcal{D}(\mathbf{T}) & =\left\{u_{1} \in H: \quad \text { there exists } \quad u_{2} \in H \quad \text { such that } \quad\left\langle u_{1}, u_{2}\right\rangle \in \mathbf{T}\right\}, \\
\mathcal{R}(\mathbf{T}) & =\left\{u_{2} \in H: \quad \text { there exists } \quad u_{1} \in H \quad \text { such that } \quad\left\langle u_{1}, u_{2}\right\rangle \in \mathbf{T}\right\}, \\
\mathbf{T}^{-1} & =\left\{\left\langle u_{2}, u_{1}\right\rangle:\left\langle u_{1}, u_{2}\right\rangle \in \mathbf{T}\right\} .
\end{aligned}
$$

An arbitrary l.r. $\mathbf{T}$ has the decomposition $\mathbf{T}=\operatorname{Gr}(T) \oplus\langle 0, \mathbf{T}(0)\rangle$, where $T$ is a linear operator (the operator part of $\mathbf{T}$ ), $\operatorname{Gr}(\mathrm{T})=\langle u, T u\rangle, u \in \mathcal{D}(T)$, and $\mathcal{D}(T)=\mathcal{D}(\mathbf{T})$. It is evident that $\mathbf{T}(u)=T u \oplus \mathbf{T}(0)$ for all $u \in \mathcal{D}(\mathbf{T})$ and $\mathcal{R}(\mathbf{T})=$ $\mathcal{R}(T) \oplus \mathbf{T}(0)$. An l.r. $\mathbf{T}$ is called Hermitian if the form $(\mathbf{T}(u), u)$ is real for all $u \in \mathcal{D}(\mathbf{T})$. If $\mathbf{T}$ is Hermitian, then the subspace $\mathbf{T}(0)$ is orthogonal to $\overline{\mathcal{D}(\mathbf{T})}$ [19]. An l.r. $\mathbf{T}$ is called self-adjoint if it is Hermitian and has no Hermitian extensions. In this case the operator part $T$ [7] is self-adjoint in the subspace $\overline{\mathcal{D}(\mathbf{T})}$ and $\mathbf{T}(0) \oplus \overline{\mathcal{D}(\mathbf{T})}=$ $H$. An l.r. $\mathbf{T}$ is called nonnegative if $(\mathbf{T}(u), u) \geq 0$ for all $u \in \mathcal{D}(\mathbf{T})$. Let $\tau[\cdot, \cdot]$ be a sesquilinear, symmetric and nonnegative form in a Hilbert space $H$ defined on a linear manifold $\mathcal{D}[\tau]$, i.e. $\tau[u, v]=\overline{\tau[v, u]}$ and $\tau[u]:=\tau[u, u] \geq 0$ for all $u, v \in \mathcal{D}[\tau]$. A sequence $\left\{u_{n}\right\}$ is called $\tau$-converging to the vector $u \in H$ if [14]

$$
\lim _{n \rightarrow \infty} u_{n}=u \text { and } \lim _{n, m \rightarrow \infty} \tau\left[u_{n}-u_{m}\right]=0 .
$$

The form $\tau$ is called closed if for every sequence $\left\{u_{n}\right\} \tau$-converging to a vector $u$, it follows that $u \in \mathcal{D}[\tau]$ and $\lim _{n \rightarrow \infty} \tau\left[u-u_{n}\right]=0$. A form $\tau$ is closed if and only if the linear manifold $\mathcal{D}[\tau]$ is a Hilbert space with the inner product $(u, v)_{\tau}=$ $\tau[u, v]+(u, v)[14$. If $\tau$ is a closed densely defined nonnegative form, then according to the first representation theorem [14] there exists a unique nonnegative self-adjoint operator $T$ in $H$, associated with $\tau$ in the following sense: $(T u, v)=\tau[u, v]$ for all $u \in \mathcal{D}(T)$ and for all $v \in \mathcal{D}[\tau]$. By the second representation theorem [14 the identities hold:

$$
\mathcal{D}[\tau]=\mathcal{D}\left(T^{1 / 2}\right), \tau[u, v]=\left(T^{1 / 2} u, T^{1 / 2} v\right), u, v \in \mathcal{D}[\tau] .
$$

If $\tau$ is a closed nonnegative but nondensely defined form, then we will associate with $\tau$ the nonnegative self-adjoint linear relation [19]

$$
\mathbf{T}=\{\langle u, T u+h\rangle, u \in \mathcal{D}(T), h \in H \ominus \overline{\mathcal{D}[\tau]}\},
$$

where $T$ is a nonnegative self-adjoint operator (the operator part of $\mathbf{T}$ ) associated with $\tau$ in the subspace $\overline{\mathcal{D}[\tau]}$. Clearly, $\mathbf{T}(0)=H \ominus \overline{\mathcal{D}[\tau]}$. The inverse linear relation $\mathbf{T}^{-1}$ is associated with the form

$$
\tau^{-1}\left[f+h_{1}, g+h_{2}\right]:=\left(\widehat{T}^{-1 / 2} f, \widehat{T}^{-1 / 2} g\right), f, g \in \mathcal{R}\left(T^{1 / 2}\right), h_{1}, h_{2} \in \mathbf{T}(0),
$$


where $\widehat{T}^{-1 / 2}=\left(T^{1 / 2} \uparrow \overline{\mathcal{R}\left(T^{1 / 2}\right)}\right)^{-1}$. We will denote by $\mathcal{R}[\mathbf{T}]$ the linear manifold $\mathcal{R}\left(T^{1 / 2}\right) \oplus \mathbf{T}(0)$. The form $\tau$ is called closable if it has a closed extension; in this case the closure of $\tau$ is the smallest closed extension of $\tau$. If $S$ is a nonnegative Hermitian operator $((S u, u) \geq 0$ for all $u \in \mathcal{D}(S))$, then the form $\tau[u, v]:=(S u, v)$ is closable. Following the notations of M.G. Kreln we denote by $S[\cdot, \cdot]$ the closure of the form $\tau$ and by $\mathcal{D}[S]$ its domain. By definition $S[u]=S[u, u]$ for all $u \in \mathcal{D}[S]$. We use the same notations for the case of a nonnegative linear relation. Note that if $\mathbf{T}$ is a nonnegative linear relation with the operator part $T$, then $\mathcal{D}[\mathbf{T}]=\mathcal{D}[T]$ and $\mathbf{T}[u, v]=T[u, v]$ for all $u, v \in \mathcal{D}[\mathbf{T}]$.

Let $\mathbf{T}_{1}$ and $\mathbf{T}_{2}$ be two nonnegative self-adjoint linear relations. We shall write $\mathbf{T}_{1} \geq \mathbf{T}_{2}$ if $\mathcal{D}\left[\mathbf{T}_{1}\right] \subseteq \mathcal{D}\left[\mathbf{T}_{2}\right]$ and $\mathbf{T}_{1}[u] \geq \mathbf{T}_{2}[u]$ for all $u \in \mathcal{D}\left[\mathbf{T}_{1}\right]$. In order to prove our main results we need the following propositions which can be proved by passing to the operator part of the l.r.

Proposition 1. Let $\mathbf{T}_{1}$ and $\mathbf{T}_{2}$ be two nonnegative self-adjoint linear relations in a Hilbert space $H$. Then the following conditions are equivalent:

1. $\mathbf{T}_{1} \geq \mathbf{T}_{2}$

2. $\mathbf{T}_{1}^{-1} \leq \mathbf{T}_{2}^{-1}$

3. $\mathcal{R}\left(\mathbf{T}_{2}\right) \subseteq \mathcal{R}\left[\mathbf{T}_{1}\right]$ and $\mathbf{T}_{1}^{-1}\left[\mathbf{T}_{2}(u)\right] \leq\left(\mathbf{T}_{2}(u), u\right)$ for all $u \in \mathcal{D}\left(\mathbf{T}_{2}\right)$.

Note that the equivalence $1 . \Longleftrightarrow 2$. is well known for linear operators [14].

Proposition 2. Let $\mathbf{T}_{1}$ and $\mathbf{T}_{2}$ be two nonnegative self-adjoint l.r.'s such that $\mathbf{T}_{1} \geq \mathbf{T}_{2}$. Then the closure of the form $\left(\mathbf{T}_{1}(f), g\right)-\mathbf{T}_{2}[f, g], f, g \in \mathcal{D}\left(\mathbf{T}_{1}\right)$, in the Hilbert space $\mathcal{D}\left[\mathbf{T}_{2}\right]$ coincides with the form

$$
\mathbf{T}_{1}[u, v]-\mathbf{T}_{2}[u, v], u, v \in \mathcal{D}\left[\mathbf{T}_{1}\right]
$$

Proof. The form $\bar{t}[u, v]:=\mathbf{T}_{1}[u, v]-\mathbf{T}_{2}[u, v], u, v \in \mathcal{D}[\bar{t}]=\mathcal{D}\left[\mathbf{T}_{1}\right]$ is nonnegative and closed in the Hilbert space $\mathcal{D}\left[\mathbf{T}_{2}\right]$. Let $u \in \mathcal{D}\left[\mathbf{T}_{1}\right]$. Then there exists a sequence $\left\{f_{n}\right\} \subset \mathcal{D}\left(\mathbf{T}_{1}\right)$ such that $\lim _{n \rightarrow \infty} f_{n}=u$ and $\lim _{m, n \rightarrow \infty}\left(\mathbf{T}_{1}\left(f_{n}-f_{m}\right), f_{n}-f_{m}\right)=0$. Then $\lim _{m, n \rightarrow \infty} \mathbf{T}_{2}\left[f_{n}-f_{m}\right]=0$, therefore, the sequence $\left\{f_{n}\right\}$ converges to $u$ in the space $\mathcal{D}\left[\mathbf{T}_{2}\right]$ and $\lim _{n \rightarrow \infty}\left\{\mathbf{T}_{1}\left[f_{n}-u\right]-\mathbf{T}_{2}\left[f_{n}-u\right]\right\}=0$. Thus, the form $\bar{t}$ is the closure of the form $\left(\mathbf{T}_{1}(f), g\right)-\mathbf{T}_{2}[f, g]$ in the Hilbert space $\mathcal{D}\left[\mathbf{T}_{2}\right]$.

3.

Let $S$ be a closed densely defined symmetric and nonnegative operator in a Hilbert space $H$ and let $S^{*}$ be its adjoint. As is well known [14], the extension $S_{F}$ of $S$ obtained by K.Friedrichs [10] is defined as a nonnegative self-adjoint extension associated with the form $S[\cdot, \cdot]$. Clearly, $\mathcal{D}\left(S_{F}\right)=\mathcal{D}[S] \cap \mathcal{D}\left(S^{*}\right), S_{F}=S^{*} \uparrow \mathcal{D}\left(S_{F}\right)$. If $\mathfrak{N}_{z}=\operatorname{Ker}\left(S^{*}-z I\right)$ are the defect subspaces, then $\mathcal{D}[S] \cap \mathfrak{N}_{z}=\{0\}, z \in \rho\left(S_{F}\right)$ and

$$
\mathcal{R}\left(S_{F}^{1 / 2}\right)=\left\{h \in H: \sup \left[|(h, f)|^{2} /(S f, f), f \in \mathcal{D}(S)\right]<\infty\right\} .
$$

In addition, $\left\|\widehat{S}_{F}^{-1 / 2} h\right\|^{2}=\sup \left[|(h, f)|^{2} /(S f, f), f \in \mathcal{D}(S)\right]$.

As was established by M.G.Kreĭn [15], 16], a nonnegative symmetric operator $S$ has a minimal nonnegative self-adjoint extension which coincides with the extension obtained by J.von Neumann [18] (in the case of a positive lower bound of $S$ ). This 
extension we call the Krĕn-von Neumann extension $S_{N}$. The operator $S_{N}$ can be defined as follows [2], 7]: $S_{N}=\left(\left(S^{-1}\right)_{F}\right)^{-1}$, where $S^{-1}$ denotes in this context the inverse l.r. to the $\operatorname{Gr}(S)$. Thus, for every nonnegative self-adjoint extension $\widetilde{S}$ of $S$, the inequality $S_{N} \leq \widetilde{S} \leq S_{F}$ holds. In addition,

$$
\begin{gathered}
\widetilde{S}[f, u]=\left(f, S^{*} u\right), f \in \mathcal{D}[S], u \in \mathcal{D}[\widetilde{S}] \cap \mathcal{D}\left[S^{*}\right] \\
\mathcal{D}[\widetilde{S}]=\mathcal{D}[S]+\mathfrak{N}_{z} \cap \mathcal{D}[\widetilde{S}]
\end{gathered}
$$

We will use the following relations (see [2]):

$$
\mathcal{D}\left[S_{N}\right]=\left\{u \in H: \sup \left[|(u, S f)|^{2} /(S f, f), f \in \mathcal{D}(S)\right]<\infty\right\}
$$

and $S_{N}[u]=\sup \left[|(u, S f)|^{2} /(S f, f), f \in \mathcal{D}(S)\right], u \in \mathcal{D}\left[S_{N}\right]$. From (44) and (11) we see the equivalence

$$
u \in \mathcal{D}\left(S^{*}\right) \cap \mathcal{D}\left[S_{N}\right] \Longleftrightarrow S^{*} u \in \mathcal{R}\left(S_{F}^{1 / 2}\right)
$$

and the equality

$$
S_{N}[u]=S_{F}^{-1}\left[S^{*} u\right], u \in \mathcal{D}\left(S^{*}\right) \cap \mathcal{D}\left[S_{N}\right]
$$

In particular,

$$
\mathfrak{N}_{z} \cap \mathcal{D}\left[S_{N}\right]=\mathfrak{N}_{z} \cap \mathcal{R}\left(S_{F}^{1 / 2}\right)
$$

and for $\varphi_{z} \in \mathfrak{N}_{z} \cap \mathcal{D}\left[S_{N}\right]$ we have

$$
S_{N}\left[\varphi_{z}\right]=|z|^{2} S_{F}^{-1}\left[\varphi_{z}\right] .
$$

From (2), (3) and (8) and the polarization identity we obtain for all $f, g \in \mathcal{D}[S]$, $\varphi_{z}, \psi_{z} \in \mathfrak{N}_{z} \cap \mathcal{D}\left[S_{N}\right]$ and $z \in \rho\left(S_{F}\right)$

$$
S_{N}\left[f+\varphi_{z}, g+\psi_{z}\right]=\left(S_{F}^{1 / 2} f+z \widehat{S}_{F}^{-1 / 2} \varphi_{z}, S_{F}^{1 / 2} g+z \widehat{S}_{F}^{-1 / 2} \psi_{z}\right) .
$$

The next theorem gives a description of all closed forms associated with nonnegative self-adjoint extensions of $S$.

Theorem 1 ([3], [5]). Let $\widetilde{S}$ be a nonnegative self-adjoint extension of $S$. Then the form $(\widetilde{S} u, v)-S_{N}[u, v], u, v \in \mathcal{D}(\widetilde{S})$, is nonnegative and closable in the Hilbert space $\mathcal{D}\left[S_{N}\right]$. Moreover, the formulas

$$
\widetilde{S}[u, v]=S_{N}[u, v]+\tau[u, v], u, v \in \mathcal{D}[\widetilde{S}]=\mathcal{D}[\tau]
$$

give a one-to-one correspondence between all closed forms $\widetilde{S}[\cdot, \cdot \cdot]$ associated with nonnegative self-adjoint extensions $\widetilde{S}$ of $S$ and all nonnegative forms $\tau[\cdot, \cdot]$ which are closed in the Hilbert space $\mathcal{D}\left[S_{N}\right]$ and such that $\tau[f]=0$ for all $f \in \mathcal{D}[S]$.

Recall that two self-adjoint extensions $\widetilde{S}_{1}$ and $\widetilde{S}_{2}$ of a symmetric operator $S$ are disjoint (relatively prime) if $\mathcal{D}\left(\widetilde{S}_{1}\right) \cap \mathcal{D}\left(\widetilde{S}_{2}\right)=\mathcal{D}(S)$ and transversal if in addition $\mathcal{D}\left(\widetilde{S}_{1}\right)+\mathcal{D}\left(\widetilde{S}_{2}\right)=\mathcal{D}\left(S^{*}\right)$. The necessary and sufficient condition for transversality of the Friedrichs and Kreın-von Neumann extensions is the following: $\mathfrak{N}_{z} \subset \mathcal{D}\left[S_{N}\right]$ for some (and then for all) $z \in \rho\left(S_{F}\right)$. This condition is equivalent to $\mathfrak{N}_{z} \subset \mathcal{R}\left[S_{F}\right]$ for

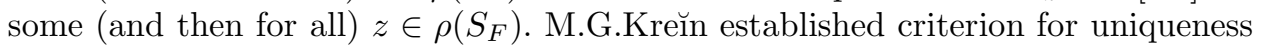
of nonnegative self-adjoint extensions of a nonnegative densely defined operator $S$. Below we present an equivalent condition for uniqueness. 
Consider the domain $\mathcal{D}\left(S^{*}\right)$ of the adjoint operator $S^{*}$ to a closed densely defined nonnegative symmetric operator $S$ as a Hilbert space $H_{+}$with the inner product $(f, g)_{+}=(f, g)+\left(S^{*} f, S^{*} g\right)$. The (+)-orthogonal decomposition holds: $H_{+}=$ $\mathcal{D}(S) \oplus \mathfrak{N}_{i} \oplus \mathfrak{N}_{-i}$. Denote

$$
\mathfrak{N}_{F}=\mathcal{D}\left(S_{F}\right) \ominus \mathcal{D}(S), \mathfrak{M}_{F}=H_{+} \ominus \mathcal{D}\left(S_{F}\right)
$$

((+)-orthogonal complements of $\mathcal{D}(S)$ in $\mathcal{D}\left(S_{F}\right)$ and of $\mathcal{D}\left(S_{F}\right)$ in $\left.H_{+}\right)$. Note that

$$
\mathfrak{M}_{F}=S_{F} \mathfrak{N}_{F}, \mathfrak{N}_{ \pm i}=\left(S_{F} \pm i I\right) \mathfrak{N}_{F}, H_{+}=\mathcal{D}(S) \oplus \mathfrak{N}_{F} \oplus \mathfrak{M}_{F}
$$

Proposition 3. A necessary and sufficient condition for the uniqueness of a nonnegative self-adjoint extension of $S$ is the equality $\mathcal{R}\left(S_{F}^{1 / 2}\right) \cap \mathfrak{N}_{F}=\{0\}$.

Suppose that

$$
\mathfrak{N}_{0}=\mathcal{R}\left(S_{F}^{1 / 2}\right) \cap \mathfrak{N}_{F} \neq\{0\} .
$$

According to Proposition 3 an operator $S$ has nonunique nonnegative self-adjoint extensions. In the following we give a parametrization of all of them. First of all we describe the closed form, associated with the KreĬn-von Neumann extension $S_{N}$ of $S$.

Proposition 4. The following equalities hold:

$$
\left\{\begin{array}{l}
\mathcal{D}\left[S_{N}\right]=\mathcal{D}[S] \dot{+} S_{F} \mathfrak{N}_{0}, \\
S_{N}\left[f+S_{F} e\right]=\left\|S_{F}^{1 / 2} f-\widehat{S}_{F}^{-1 / 2} e\right\|^{2}, f \in \mathcal{D}[S], e \in \mathfrak{N}_{0} .
\end{array}\right.
$$

Proof. From $S_{F} \geq 0$ and the equality $S^{*} S_{F} e=-e$ for all $e \in \mathfrak{N}_{F}$ it follows that $\mathcal{D}[S] \cap S_{F} \mathfrak{N}_{F}=\{0\}$. From $\mathfrak{N}_{i}=\left(S_{F}+i I\right) \mathfrak{N}_{F}$, (3) and (7), we have $\mathcal{D}\left[S_{N}\right]=$ $\mathcal{D}[S]+\mathcal{R}\left(S_{F}^{1 / 2}\right) \cap \mathfrak{N}_{i}$. Hence, we get that $\mathcal{D}\left[S_{N}\right]=\mathcal{D}[S]+S_{F} \mathfrak{N}_{0}$. From (9) it follows for all $f \in \mathcal{D}[S]$ and all $e \in \mathfrak{N}_{0}$ that

$$
\begin{aligned}
& S_{N}\left[f+S_{F} e\right]=S_{N}\left[f-i e+\left(S_{F}+i I\right) e\right] \\
& =\left\|S_{F}^{1 / 2}(f-i e)+i \widehat{S}_{F}^{-1 / 2}\left(S_{F}+i I\right) e\right\|^{2}=\left\|S_{F}^{1 / 2} f-\widehat{S}_{F}^{-1 / 2} e\right\|^{2} .
\end{aligned}
$$

Proposition 5. Suppose that condition (10) is fulfilled and define a nonnegative sesquilinear form

$$
w_{0}[e, g]=\left(S_{F}^{1 / 2} e, S_{F}^{1 / 2} g\right)+\left(\widehat{S}_{F}^{-1 / 2} e, \widehat{S}_{F}^{-1 / 2} g\right), e, g \in \mathfrak{N}_{0} .
$$

Then the form $w_{0}$ is closed in the Hilbert space $H_{+}$and

$$
w_{0}[e] \geq 2\|e\|^{2} \text { for all } e \in \mathfrak{N}_{0} .
$$

Denote by $\mathfrak{N}_{0}^{w_{0}}$ the Hilbert space with the inner product

$$
(e, h)_{w_{0}}=w_{0}[e, h]+(e, h)_{+}, e, h \in \mathfrak{N}_{0} .
$$

Then the operator $S_{F}$ is an isomorphism of the Hilbert space $\mathfrak{N}_{0}^{w_{0}}$ and the subspace $S_{F} \mathfrak{N}_{0}$ of the Hilbert space $\mathcal{D}\left[S_{N}\right]$. 
Proof. From (12) it follows that

$$
w_{0}[e]=\left\|S_{F}^{1 / 2} e-\widehat{S}_{F}^{-1 / 2} e\right\|^{2}+2\|e\|^{2}=\left\|S_{F}^{1 / 2} e \pm i \widehat{S}_{F}^{-1 / 2} e\right\|^{2} .
$$

Therefore, $w_{0}[e] \geq 2\|e\|^{2}$ for all $e \in \mathfrak{N}_{0}$. Let $\lim _{n \rightarrow \infty} e_{n}=e$ in $H_{+}$, and let $\lim _{n, m \rightarrow \infty} w_{0}\left[e_{n}-e_{m}\right]=0$. Then $\lim _{n \rightarrow \infty} e_{n}=e$ in $H$ and $\left\{S_{F}^{1 / 2} e_{n}\right\},\left\{\widehat{S}_{F}^{-1 / 2} e_{n}\right\}$ are Cauchy sequences. Since the operators $S_{F}^{1 / 2}$ and $\widehat{S}_{F}^{-1 / 2}$ are closed, we get that

$$
S_{F}^{1 / 2} e=\lim _{n \rightarrow \infty} S_{F}^{1 / 2} e_{n}, e \in \mathcal{R}\left(S_{F}^{1 / 2}\right) \quad \text { and } \quad \widehat{S}_{F}^{-1 / 2} e=\lim _{n \rightarrow \infty} S_{F}^{-1 / 2} e_{n} .
$$

Thus $e \in \mathfrak{N}_{0}$ and $\lim _{n \rightarrow \infty} w_{0}\left[e-e_{n}\right]=0$. This implies that the form $w_{0}$ is closed in $H_{+}$. From the inequality $\left\|\Lambda^{1-\theta} u\right\| \leq\|\Lambda u\|^{1-\theta}\|u\|^{\theta}$ for all $u \in \mathcal{D}(\Lambda)$, which is true for an arbitrary nonnegative self-adjoint operator $\Lambda$ and an arbitrary $\theta \in[0,1]$, we obtain, for all $e \in \mathcal{D}\left(S_{F}\right) \cap \mathcal{R}\left(S_{F}^{1 / 2}\right)$,

$$
\|e\| \leq\left\|S_{F} e\right\|^{1 / 3}\left\|\widehat{S}_{F}^{-1 / 2} e\right\|^{2 / 3},\left\|S_{F}^{1 / 2} e\right\| \leq\left\|S_{F} e\right\|^{2 / 3}\left\|\widehat{S}_{F}^{-1 / 2} e\right\|^{1 / 3} .
$$

Therefore, statement 2. follows from (10) and (11).

Let $\mathbf{W}_{0}$ be a $(+)$-nonnegative self-adjoint linear relation in $\mathfrak{N}_{F}$ associated with $w_{0}$. In view of $w_{0}[f]>0$ for all $f \neq 0 \in \mathfrak{N}_{0}$, the inverse l.r. $\mathbf{W}_{0}^{-1}$ is densely defined in $\mathfrak{N}_{F}$ and therefore is the graph of a $(+)$-self-adjoint nonnegative operator. We denote this operator by $W_{0}^{-1}$. Clearly, $\operatorname{Ker} W_{0}^{-1}=\mathbf{W}(0)=\mathfrak{N}_{F} \ominus \mathfrak{N}_{0}($ the $(+)$ orthogonal complement).

The next theorem gives a description of all nonnegative self-adjoint extensions of $S$ and their associated closed forms in terms of $W_{0}^{-1}$ and some auxiliary operators in $\mathfrak{N}_{F}$.

Theorem 2. The formulas

$$
\begin{aligned}
& \left\{\begin{array}{l}
\mathcal{D}(\widetilde{S})=\mathcal{D}(S) \oplus\left(I+S_{F} \widetilde{U}\right) \mathcal{D}(\widetilde{U}), \\
\widetilde{S}\left(f_{0}+e+S_{F} \widetilde{U} e\right)=S_{F}\left(f_{0}+e\right)-\widetilde{U} e, f_{0} \in \mathcal{D}(S), e \in \mathcal{D}(\widetilde{U}),
\end{array}\right. \\
& \left\{\begin{array}{c}
\mathcal{D}[\widetilde{S}]=\mathcal{D}[S]+S_{F} \mathcal{R}\left(\widetilde{U}^{1 / 2}\right), \\
\widetilde{S}\left[f+S_{F} h\right]=\left\|S_{F}^{1 / 2} f-S_{F}^{-1 / 2} h\right\|^{2}+\widetilde{U}^{-1}[h]-w_{0}[h], \\
f \in \mathcal{D}[S], h \in \mathcal{R}\left(\widetilde{U}^{1 / 2}\right)
\end{array}\right.
\end{aligned}
$$

give a one-to-one correspondence between all nonnegative self-adjoint extensions $\widetilde{S}$ of $S$, their associated closed forms and all $(+)$-nonnegative self-adjoint operators $\widetilde{U}$ in $\mathfrak{N}_{F}$ satisfying the condition

$$
\widetilde{U} \leq W_{0}^{-1} .
$$

An extension $\widetilde{S}$ coincides with $S_{N}$ if $\widetilde{U}=W_{0}^{-1}$. The extensions $S_{F}$ and $S_{N}$ are disjoint if and only if $\mathfrak{N}_{0}$ is dense in $\mathfrak{N}_{F}$, and are transversal if and only if $\mathfrak{N}_{0}=\mathfrak{N}_{F}$.

Proof. For every $h \in \mathfrak{N}_{F}$ we have $\left(S^{*} S_{F} h, S_{F} h\right)=-\left(h, S_{F} h\right) \leq 0$. It follows that, if $\widetilde{S}=\widetilde{S}^{*}$ is a nonnegative extension of $S$, then $\mathcal{D}(\widetilde{S}) \cap S_{F} \mathfrak{N}_{F}=\{0\}$. Therefore, we obtain $\mathcal{D}(\widetilde{S})=\mathcal{D}(S) \oplus\left(I+S_{F} \widetilde{U}\right) \mathcal{D}(\widetilde{U})$, where $\widetilde{U}$ is a closed linear operator in the subspace $\mathfrak{N}_{F}$ with the domain $\mathcal{D}(\widetilde{U})$. Let us show that $\widetilde{U}$ is a (+)-self-adjoint 
operator in $\mathfrak{N}_{F}$ and satisfies condition (15). Consider an arbitrary vector $f \in \mathcal{D}(\widetilde{S})$ of the form $f=h+S_{F} \widetilde{U} h, h \in \mathcal{D}(\widetilde{U})$. Then $\widetilde{S} f=S^{*} f=S_{F} h-\widetilde{U} h$ and

$$
\begin{aligned}
& (\widetilde{S} f, f)=\left(S_{F} h-\widetilde{U} h, h+S_{F} \widetilde{U} h\right) \\
& =\left(S_{F} h, h\right)-\left(\widetilde{U} h, S_{F} \widetilde{U} h\right)+(h, \widetilde{U} h)_{+}-2 \operatorname{Re}(\widetilde{U} h, h) .
\end{aligned}
$$

Since $\operatorname{Im}(\widetilde{S} f, f)=0$, we get that $\operatorname{Im}(h, \widetilde{U} h)_{+}=0$. This means that $\widetilde{U}$ is a $(+)$ symmetric operator in $\mathfrak{N}_{F}$. Since $\widetilde{S}$ is a nonnegative self-adjoint extension of $S$, then for every vector $f \in \mathcal{D}(\widetilde{S})$ the inequality $(\widetilde{S} f, f) \geq S_{N}[f]$ holds. Again let $f=h+S_{F} \widetilde{U} h, h \in \mathcal{D}(\widetilde{U})$. Then (5) implies $\widetilde{U} h \in \mathcal{R}\left(S_{F}^{1 / 2}\right)$ for all $h \in \mathcal{D}(\widetilde{U})$ and from (6) we obtain

$$
S_{N}[f]=\left(S_{F} h, h\right)+\left\|\widehat{S}_{F}^{-1 / 2} \widetilde{U} h\right\|^{2}-2 \operatorname{Re}(h, \widetilde{U} h) .
$$

Thus, the inequality $(\widetilde{S} f, f) \geq S_{N}[f]$ yields

$$
\begin{aligned}
\left(S_{F} h, h\right) & -\left(\widetilde{U} h, S_{F} \widetilde{U} h\right)+(h, \widetilde{U} h)_{+}-2 \operatorname{Re}(\widetilde{U} h, h) \\
\geq & \left(S_{F} h, h\right)+\left\|S_{F}^{-1 / 2} \widetilde{U} h\right\|^{2}-2 \operatorname{Re}(h, \widetilde{U} h) .
\end{aligned}
$$

Finally we have $\left\|\widehat{S}_{F}^{-1 / 2} \widetilde{U} h\right\|^{2}+\left(\widetilde{U} h, S_{F} \widetilde{U} h\right) \leq(h, \widetilde{U} h)_{+}$. It follows that $w_{0}[\widetilde{U} h] \leq$ $(\widetilde{U} h, h)_{+}$for all $h \in \mathcal{D}(\widetilde{U})$.

In particular, $\widetilde{U}$ is a $(+)$-nonnegative operator in $\mathfrak{N}_{F}$. It follows that $\widetilde{U}$ has a (+)-self-adjoint extension $\widetilde{\widetilde{U}}$ in $\mathfrak{N}_{F}$. One can check that the operator $\widetilde{\widetilde{S}}$ given by $\mathcal{D}(\widetilde{\widetilde{S}})=\mathcal{D}(S) \oplus\left(I+S_{F} \widetilde{\widetilde{U}}\right) \mathcal{D}(\widetilde{\widetilde{U}}), \widetilde{\widetilde{S}}=S^{*} \mid \mathcal{D}(\widetilde{\widetilde{S}})$ is a symmetric extension of $\widetilde{S}$ in $H$. Since $\widetilde{S}$ is self-adjoint, we get $\widetilde{\widetilde{U}}=\widetilde{U}$. Thus, $\widetilde{U}$ is a $(+)$-self-adjoint and nonnegative operator in $\mathfrak{N}_{F}$. According to Proposition 1 the inequality $w_{0}[\widetilde{U} h] \leq(\widetilde{U} h, h)_{+}$, $h \in \mathcal{D}(\widetilde{U})$, is equivalent to $\widetilde{U} \leq W_{0}^{-1}$.

Conversely, let $\widetilde{U}$ be a $(+)$-self-adjoint nonnegative operator in $\mathfrak{N}_{F}$ satisfying the condition $\widetilde{U} \leq W_{0}^{-1}$. Then also $w_{0}[\widetilde{U} h] \leq(\widetilde{U} h, h)_{+}$for all $h \in \mathcal{D}(\widetilde{U})$. It follows that

$$
\left(S^{*} f, f\right) \geq S_{N}[f] \text { for all } f=h+S_{F} h, h \in \mathcal{D}(\widetilde{U}),
$$

and from (4) we obtain $|(S \varphi, f)|^{2} \leq(S \varphi, \varphi)\left(S^{*} f, f\right)$. Hence,

$$
2 \operatorname{Re}(S \varphi, f) \geq-(S \varphi, \varphi)-\left(S^{*} f, f\right) .
$$

Further for $g=\varphi+f$, where $\varphi \in \mathcal{D}(S)$, we have

$$
\left(S^{*} g, g\right)=\left(S \varphi+S^{*} f, \varphi+f\right)=(S \varphi, \varphi)+\left(S^{*} f, f\right)+2 \operatorname{Re}(S \varphi, f) \geq 0 .
$$

Thus, the operator $\widetilde{S}=S^{*} \mid\left(\mathcal{D}(S) \oplus\left(I+S_{F} \widetilde{U}\right) \mathcal{D}(\widetilde{U})\right)$ is a self-adjoint nonnegative extension of $S$. Define a nonnegative self-adjoint extension

$$
\widetilde{S}_{0}=S^{*} \uparrow\left(\mathcal{D}(S) \oplus\left(I+S_{F} W_{0}^{-1}\right) \mathfrak{N}_{0}\right) .
$$

Let us prove that $\widetilde{S}_{0}$ coincides with the Kreĭn-von Neumann extension $S_{N}$. We will show the equality $\left(\widetilde{S}_{0} u, v\right)=S_{N}[u, v]$ for all $u \in \mathcal{D}\left(\widetilde{S}_{0}\right)$ and all $v \in \mathcal{D}\left[S_{N}\right]$. Let $u=$ $f+\left(I+S_{F} W_{0}^{-1}\right) e, v=g+S_{F} h$, where $f \in \mathcal{D}(S), e \in \mathcal{D}\left(W_{0}^{-1}\right), g \in \mathcal{D}[S], h \in \mathfrak{N}_{0}$. 
Then from (11)

$$
\begin{aligned}
S_{N}[u, v]= & \left(S_{F}^{1 / 2}(f+e)-\widehat{S}_{F}^{-1 / 2} W_{0}^{-1} e, S_{F}^{1 / 2} g-\widehat{S}_{F}^{-1 / 2} h\right) \\
= & \left(S_{F}(f+e)-W_{0}^{-1} e, g\right)-(f+e, h)+\left(\widehat{S}_{F}^{-1 / 2} W_{0}^{-1} e, \widehat{S}_{F}^{-1 / 2} h\right) \\
= & \left(S_{F}(f+e)-W_{0}^{-1} e, g\right)+\left(S_{F}(f+e), S_{F} h\right)-(f+e, h)_{+} \\
& \quad+\left(\widehat{S}_{F}^{-1 / 2} W_{0}^{-1} e, \widehat{S}_{F}^{-1 / 2} h\right)=\left(S_{F}(f+e)-W_{0}^{-1} e, g+S_{F} h\right)-(e, h)_{+} \\
& \quad+\left(\widehat{S}_{F}^{-1 / 2} W_{0}^{-1} e, \widehat{S}_{F}^{-1 / 2} h\right)+\left(W_{0}^{-1} e, S_{F} h\right) \\
= & \left(\widetilde{S}_{0} u, v\right)-(e, h)_{+}+\left(\widehat{S}_{F}^{-1 / 2} W_{0}^{-1} e, \widehat{S}_{F}^{-1 / 2} h\right)+\left(W_{0}^{-1} e, S_{F} h\right) .
\end{aligned}
$$

Put $q=W_{0}^{-1} e$. Then $e \in \mathbf{W}_{0}(q)$ and $\left(\mathbf{W}_{0}(q), h\right)_{+}=(e, h)_{+}$for all $h \in \mathcal{D}\left[w_{0}\right]=$ $\mathfrak{N}_{0}$. By the definition of the form $w_{0}$ we have

$$
\begin{aligned}
& \left(\widehat{S}_{F}^{-1 / 2} W_{0}^{-1} e, \widehat{S}_{F}^{-1 / 2} g\right)=w_{0}[q, h]-\left(S_{F} q, h\right) \\
& \quad=\left(\mathbf{W}_{0}(q), h\right)_{+}-\left(S_{F} q, h\right)=(e, h)_{+}-\left(S_{F} W_{0}^{-1} e, h\right) .
\end{aligned}
$$

Thus, $\left(\widetilde{S}_{0} u, v\right)=S_{N}[u, v]$. In accordance with the first representation theorem we obtain $\widetilde{S}_{0}=S_{N}$.

Let us prove (14). Let $u=f_{1}+S_{F} h_{1}, v=f_{2}+S_{F} h_{2}$, where $f_{1}, f_{2} \in \mathcal{D}[S]$, $h_{1}, h_{2} \in \mathcal{R}\left(\widetilde{U}^{1 / 2}\right)$. Define the form $\tau[u, v]$ in $\mathcal{D}\left[S_{N}\right]$ :

$$
\tau[u, v]=\widetilde{U}^{-1}\left[h_{1}, h_{2}\right]-w_{0}\left[h_{1}, h_{2}\right] .
$$

Proposition 1 implies nonnegativity of $\tau$. According to Proposition 2, the form $\widetilde{U}^{-1}\left[h_{1}, h_{2}\right]-w_{0}\left[h_{1}, h_{2}\right]$ is closed in the Hilbert space $\mathfrak{N}_{0}^{w_{0}}=\mathcal{D}\left[w_{0}\right]$ and is the closure of the form $\left(e_{1}, \widetilde{U} e_{2}\right)_{+}-w_{0}\left[\widetilde{U} e_{1}, \widetilde{U} e_{2}\right], e_{1}, e_{2} \in \mathcal{D}(\widetilde{U})$. Using Propositions 4 and 5 we get that $\tau$ is closed in the Hilbert space $\mathcal{D}\left[S_{N}\right]$.

For $u=\varphi_{1}+\left(I+S_{F} \widetilde{U}\right) e_{1}, v=\varphi_{2}+\left(I+S_{F} \widetilde{U}\right) e_{2}, \varphi_{1}, \varphi_{2} \in \mathcal{D}(S), e_{1}, e_{2} \in \mathcal{D}(\widetilde{U})$ from (13) and (11) we have the equality

$$
(\widetilde{S} u, v)-S_{N}[u, v]=\left(e_{1}, \widetilde{U} e_{2}\right)_{+}-w_{0}\left[\widetilde{U} e_{1}, \widetilde{U} e_{2}\right] .
$$

Therefore, Theorem 1 yields equalities (14).

As a consequence of this theorem we get

Theorem 3. Let $P_{i}^{+}$be the orthogonal projection onto $\mathfrak{N}_{i}$ in $H_{+}$and $\mathcal{D}\left(S_{F}\right)=$ $\mathcal{D}(S) \dot{+}\left(I+V_{F}\right) \mathfrak{N}_{i}$. Then the operator

$$
\widetilde{V} P_{i}^{+} h=-V_{F} P_{i}^{+}(\widetilde{U}+i I)(\widetilde{U}-i I)^{-1} h, h \in \mathfrak{N}_{F},
$$

defines $\mathcal{D}(\widetilde{S})$ by the von Neumann formula $\mathcal{D}(\widetilde{S})=\mathcal{D}(S) \dot{+}(I+\widetilde{V}) \mathfrak{N}_{i}$, where $\widetilde{U}$ is $a(+)$-self-adjoint operator in $\mathfrak{N}_{F}$ satisfying the condition $0 \leq \widetilde{U} \leq W_{0}^{-1}$.

Proof. Let $\widetilde{U}$ be a $(+)$-selfadjoint operator in the subspace $\mathfrak{N}_{F}$ which satisfies the condition $0 \leq \widetilde{U} \leq W_{0}^{-1}$ and let $\widetilde{Z}=(\widetilde{U}+i I)(\widetilde{U}-i I)^{-1}$ be the Cayley transform 
of $\widetilde{U}$. Then $\widetilde{Z}$ is a $(+)$-unitary operator in $\mathfrak{N}_{F}$ and $\widetilde{U}$ can be defined as follows: $e=i(I-\widetilde{Z}) h, \widetilde{U} e=(I+\widetilde{Z}) h, h \in \mathfrak{N}_{F}$. Since $\mathfrak{N}_{F}=\left(I+V_{F}\right) \mathfrak{N}_{i}$ and $V_{F}$ is a (+)-isometry from $\mathfrak{N}_{i}$ onto $\mathfrak{N}_{-i}$, we get that if $h=\left(I+V_{F}\right) g, g \in \mathfrak{N}_{i}$, then $\widetilde{Z} h=\left(I+V_{F}\right) \widetilde{K} g$, where $\widetilde{K}$ is a $(+)$-unitary operator in $\mathfrak{N}_{i}$. This implies that $P_{i}^{+} h=g, P_{i}^{+} \widetilde{Z} h=\widetilde{K} g$, and $e=i\left(I+V_{F}\right)(g-\widetilde{K} g), \widetilde{U} e=\left(I+V_{F}\right)(g+\widetilde{K} g)$, $S_{F} \widetilde{U} e=\left(I-V_{F}\right)(g+\widetilde{K}) g$,

$$
\begin{aligned}
\left(I+S_{F} \widetilde{U}\right) e & =i\left(I+V_{F}\right)(g-\widetilde{K} g)+i\left(I-V_{F}\right)(g+\widetilde{K} g) \\
& =2 i g-2 i V_{F} \widetilde{K} g .
\end{aligned}
$$

Hence, for a self-adjoint extension $\widetilde{S}$ given by (13) we get

$$
\mathcal{D}(\widetilde{S})=\mathcal{D}(S) \dot{+}\left(I-V_{F} \widetilde{K}\right) \mathfrak{N}_{i}
$$

5.

Let $y \in \mathbb{R}^{3}$. Consider the operator $S$ defined as follows:

$$
\mathcal{D}(S)=\left\{\varphi(x) \in H_{2}^{2}\left(\mathbb{R}^{3}\right): \varphi(y)=0\right\}, \quad S \varphi=-\Delta \varphi,
$$

where $x \in \mathbb{R}^{3}, H_{2}^{2}\left(\mathbb{R}^{3}\right)$ is the Sobolev space and $\Delta$ denotes the Laplacian. As is well known the operator $S$ is a nonnegative symmetric operator in $L^{2}\left(\mathbb{R}^{3}, d x\right)$ with defect numbers 1,1 and its Friedrichs extension $S_{F}$ is given by $\mathcal{D}\left(S_{F}\right)=H_{2}^{2}\left(\mathbb{R}^{3}\right), S_{F}=$ $-\Delta$.

Let $\mathcal{F}: L^{2}\left(\mathbb{R}^{3}, d x\right) \rightarrow L^{2}\left(\mathbb{R}^{3}, d p\right)$,

$$
\mathcal{F} f=\widehat{f}(p)=s-\lim _{R \rightarrow \infty}(2 \pi)^{-3 / 2} \int_{|x| \leq R} f(x) \exp (-i x p) d x, \quad p=\left(p_{1}, p_{2}, p_{3}\right),
$$

be the Fourier transform. In the $p$-representation we obtain the nonnegative symmetric operator $\stackrel{\circ}{\AA}$ and its Friedrichs extension $A_{F}$ :

$$
\begin{aligned}
& \mathcal{D}(\stackrel{\circ}{A})=\left\{h(p) \in L^{2}\left(\mathbb{R}^{3}, d p\right), \int_{\mathbb{R}^{3}} h(p) \exp (i p y) d p=0\right\}, \\
& \mathcal{D}\left(A_{F}\right)=H_{2}\left(\mathbb{R}^{3}\right):=L^{2}\left(\mathbb{R}^{3},\left(|p|^{4}+1\right) d p\right), \\
& \stackrel{\circ}{A} h=|p|^{2} h(p), h(p) \in \mathcal{D}(A), \\
& A_{F} f=|p|^{2} f(p), f(p) \in \mathcal{D}\left(A_{F}\right) .
\end{aligned}
$$

Let $e(p)=\exp (-i p y)\left(1+|p|^{4}\right)^{-1}$. Clearly, $\mathfrak{N}_{F}=\operatorname{span}\{e(p)\}, \mathfrak{M}_{F}=A_{F} \mathfrak{N}_{F}=$ $\operatorname{span}\left\{|p|^{2} e(p)\right\}$. The adjoint operator $\stackrel{\circ *}{A}$ is given by the following relations:

$$
\begin{aligned}
& \mathcal{D}(\stackrel{\circ *}{A})=\mathcal{D}(\stackrel{\circ}{A}) \dot{+} \mathfrak{N}_{F} \dot{+} \mathfrak{M}_{F}=H_{2}\left(\mathbb{R}^{3}\right) \dot{+} \mathfrak{M}_{F}, \\
& \stackrel{\circ}{A}^{*}\left(f(p)+\lambda|p|^{2} e(p)\right)=|p|^{2} f(p)-\lambda e(p), f(p) \in H_{2}\left(\mathbb{R}^{3}\right), \lambda \in \mathbb{C} .
\end{aligned}
$$


Let $H_{+}=\mathcal{D}(\stackrel{\circ *}{A})$. Since

$$
\mathcal{D}\left(A_{F}^{1 / 2}\right)=H_{1}\left(\mathbb{R}^{3}\right):=L^{2}\left(\mathbb{R}^{3},\left(|p|^{2}+1\right) d p\right), A_{F}^{1 / 2} f=|p| f(p),
$$

we obtain that

$$
A_{F}^{-1 / 2} e(p)=\frac{\exp (-i p y)}{|p|\left(1+|p|^{4}\right)} \in H_{1}\left(\mathbb{R}^{3}\right)
$$

By Proposition 3 we have $A_{F} \neq A_{N}$. By the direct calculation we get

$$
\begin{aligned}
& (e(p), e(p))_{+}=\int_{\mathbb{R}^{3}} \frac{d p}{1+|p|^{4}}=\sqrt{2} \pi^{2}, \\
& \left(A_{F}^{1 / 2} e(p), A_{F}^{1 / 2} e(p)\right)+\left(A_{F}^{-1 / 2} e(p), A_{F}^{-1 / 2} e(p)\right) \\
& \quad=\int_{\mathbb{R}^{3}} \frac{d p}{|p|^{2}\left(1+|p|^{4}\right)}=\sqrt{2} \pi^{2} .
\end{aligned}
$$

From Theorem 2 we get the following descriptions of non-negative self-adjoint extensions of $\stackrel{\circ}{A}$ :

$$
\begin{aligned}
& \mathcal{D}\left(A_{N}\right)=\left\{f_{0}(p)+\lambda \frac{\left(1+|p|^{2}\right) \exp (-i p y)}{1+|p|^{4}}, f_{0}(p) \in \mathcal{D}(A), \lambda \in \mathbb{C}\right\}, \\
& A_{N}\left(f_{0}(p)+\lambda \frac{\left(1+|p|^{2}\right) \exp (-i p y)}{1+|p|^{4}}\right)=|p|^{2} f_{0}(p)+\lambda \frac{\left(|p|^{2}-1\right) \exp (-i p y)}{1+|p|^{4}}, \\
& \mathcal{D}\left(\widetilde{A}_{u}\right)=\left\{f_{0}(p)+\lambda \frac{\left(1+u|p|^{2}\right) \exp (-i p y)}{1+|p|^{4}}, f_{0}(p) \in \mathcal{D}(A), \lambda \in \mathbb{C}\right\}, \\
& \widetilde{A}_{u}\left(f_{0}(p)+\lambda \frac{\left(1+u|p|^{2}\right) \exp (-i p y)}{1+|p|^{4}}\right)=|p|^{2} f_{0}(p)+\lambda \frac{\left(|p|^{2}-u\right) \exp (-i p y)}{1+|p|^{4}},
\end{aligned}
$$

where $0 \leq u \leq 1$. The inverse Fourier transform $\mathcal{F}^{-1}$ is given by the equality

$$
\mathcal{F}^{-1} \widehat{f}=f(x)=s-\lim _{R \rightarrow \infty}(2 \pi)^{-3 / 2} \int_{|p| \leq R} \widehat{f}(p) \exp (i p x) d p .
$$

We have $S=\mathcal{F}^{-1} \stackrel{\circ}{A} \mathcal{F}, S_{F}=\mathcal{F}^{-1} A_{F} \mathcal{F}, S_{N}=\mathcal{F}^{-1} A_{N} \mathcal{F}$. A calculation gives

$$
\begin{aligned}
& \mathcal{F}^{-1} e(p)=\sqrt{\frac{\pi}{2}} \frac{\exp \left(-\frac{|x-y|}{\sqrt{2}}\right)}{|x-y|} \sin \frac{|x-y|}{\sqrt{2}}, \\
& \mathcal{F}^{-1} A_{F} e(p)=\sqrt{\frac{\pi}{2}} \frac{\exp \left(-\frac{|x-y|}{\sqrt{2}}\right)}{|x-y|} \cos \frac{|x-y|}{\sqrt{2}} .
\end{aligned}
$$


Hence,

$$
\begin{aligned}
& \mathcal{D}\left(S_{N}\right)=\left\{f_{0}(x)+\lambda \frac{\exp \left(-\frac{|x-y|}{\sqrt{2}}\right)}{|x-y|}\left(\sin \frac{|x-y|}{\sqrt{2}}+\cos \frac{|x-y|}{\sqrt{2}}\right), f_{0}(y)=0, \lambda \in \mathbb{C}\right\}, \\
& S_{N}\left(f_{0}(x)+\lambda \frac{\exp \left(-\frac{|x-y|}{\sqrt{2}}\right)}{|x-y|}\left(\sin \frac{|x-y|}{\sqrt{2}}+\cos \frac{|x-y|}{\sqrt{2}}\right)\right) \\
& =-\Delta f_{0}(x)+\lambda \frac{\exp \left(-\frac{|x-y|}{\sqrt{2}}\right)}{|x-y|}\left(\cos \frac{|x-y|}{\sqrt{2}}-\sin \frac{|x-y|}{\sqrt{2}}\right), \\
& \mathcal{D}\left(\widetilde{S}_{u}\right)=\left\{f_{0}(x)+\lambda \frac{\exp \left(-\frac{|x-y|}{\sqrt{2}}\right)}{|x-y|}\left(\sin \frac{|x-y|}{\sqrt{2}}+u \cos \frac{|x-y|}{\sqrt{2}}\right), f_{0}(y)=0, \lambda \in \mathbb{C}\right\}, \\
& \widetilde{S}_{u}\left(f_{0}(x)+\lambda \frac{\exp \left(-\frac{|x-y|}{\sqrt{2}}\right)}{|x-y|}\left(\sin \frac{|x-y|}{\left.\left.\sqrt{2}+u \cos \frac{|x-y|}{\sqrt{2}}\right)\right)}\right.\right. \\
& =-\Delta f_{0}(x)+\lambda \frac{\exp \left(-\frac{|x-y|}{\sqrt{2}}\right)}{|x-y|}\left(\cos \frac{|x-y|}{\sqrt{2}}-u \sin \frac{|x-y|}{\sqrt{2}}\right), 0 \leq u \leq 1 .
\end{aligned}
$$

\section{ACKNOWLEDGMENTS}

The authors thank Fritz Gesztesy and Konstantin Makarov for valuable discussions and the referee for some remarks. Special thanks go to Joseph Ball for supplying us with numerous helpful suggestions, both mathematical and linguistic.

\section{REFERENCES}

[1] A.Alonso, B.Simon, The Birman - Krein - Vishik theory of selfadjoint extensions of semibounded operators, J. Operator Theory, 4 (1980), 251-270. MR 81m:47038

[2] T.Ando, K.Nishio, Positive selfadjoint extensions of positive symmetric operators, Tohóku Math. J., 22 (1970), 65-75. MR 41:9016

[3] Yu.M.Arlinskiı̌, Maximal sectorial extensions and closed forms associated with them, Ukrainian Mat.J. 48, No.6 (1996), 723-739 (Russian). English translation, pp. 809-827 (1997). MR 97k:47022

[4] Yu.M.Arlinskiı̌, Extremal extensions of sectorial linear relations, Matematychnii Studii, 7, No.1 (1997), 81-96. MR 2000c:47017

[5] Yu.Arlinskiı̌, S.Hassi, Z.Sebestyen, H.de Snoo, On the class of extremal extensions of a nonnegative operators, Oper. Theory Adv. Appl., 127 (2001), 41-81.

[6] M.S.Birman, On the self-adjoint extensions of positive definite operators, Mat.Sbornik 38 (1956), 431-450 (Russian).

[7] E.A.Coddington, H.S.V.de Snoo, Positive self-adjoint extensions of positive symmetric subspaces, Math. Z., 159 (1978), 203-214. MR 58:17936

[8] V.A.Derkach, M.M.Malamud, Generalized resolvents and the boundary value problems for Hermitian operators with gaps, J. Funct. Anal., 95, No.1 (1991), 1-95. MR 93d:47046

[9] V.A.Derkach, M.M.Malamud, E.R.Tsekanovskiı̌, Sectorial extensions of positive operator, Ukrainian Mat.J. 41, No.2 (1989), 151-158 (Russian). MR 90f:47005

[10] K.Friedrichs, Spektraltheorie halbbeschrankter operatoren, Math. Ann., 109 (1934), 465487.

[11] F.Gesztesy, N.Kalton, K.Makarov, E.Tsekanovskiı̌, Some applications of operator-valued Herglotz functions, Oper.Theory, Adv. and Appl., 123 (2001), 271-321. MR 2002f:47049

[12] F.Gesztesy, E.Tsekanovskiǔ, On matrix-valued Herglotz functions, Math.Nachr. 218 (2000), 61-138. MR 2001j:47018 
[13] M.L.Gorbachuk, V.I.Gorbachuk, A.N.Kochubeŭ, Extension theory for symmetric operators and boundary value problems for differential equations, Ukrainian Mat.J. 41, No.10 (1989), 1298-1313 (Russian). English translation, pp. 1117-1129.

[14] T.Kato, Perturbation theory for linear operators, Springer-Verlag, 1966.

[15] M.G.Kreı̆n, The theory of selfadjoint extensions of semibounded Hermitian transformations and its applications, I, Mat.Sbornik 20, No.3 (1947), 431-495 (Russian).

[16] M.G.Krein, The theory of selfadjoint extensions of semibounded Hermitian transformations and its applications, II, Mat.Sbornik 21, No.3 (1947), 365-404 (Russian).

[17] V.E.Lyantse, H.B.Majorga, On selfadjoint extensions of Schrödinger operator with a singular potential. Lviv university. Deposited in VINITI 15.01.81, N 240-81DEP.

[18] J.von Neumann, Allgemeine eigenwerttheorie Hermitescher funktionaloperatoren, Math. Ann. 102 (1929), 49-131.

[19] F.S.Rofe-Beketov, Numerical range of a linear relation and maximal relations, Theory of Functions, Functional Anal. and Appl., No.44 (1985), 103-112 (Russian). MR 86m:47004

[20] M.I.Vishik, On general boundary conditions for elliptic differential equations, Trudy Moskov. Mat.Obsc., 1 (1952), 187-246 (Russian). Amer.Math.Soc.Transl. (2), 24 (1963), 107-172.

Department of Mathematics, East Ukrainian National University, Kvartal MolodyOZhNy, 20-A, 91034, Lugansk, Ukraine

E-mail address: yma@snu.edu.ua

Department of Mathematics, P.O. Box 2044, Niagara University, New York 14109

E-mail address: tsekanov@niagara.edu 